\title{
Erratum: Chiral state conversion without encircling an exceptional point [Phys. Rev. A 96, 052129 (2017)]
}

\author{
Absar U. Hassan, Gisela L. Galmiche, Gal Harari, Patrick LiKamWa, Mercedeh Khajavikhan, \\ Mordechai Segev, and Demetrios N. Christodoulides \\ (Received 7 December 2017; published 26 December 2017)
}

DOI: 10.1103/PhysRevA.96.069908

The numerical precision of the iterative algorithm (Runge-Kutta) was identified as insufficient when obtaining the results that were presented in Figs. 1(a)-1(c) and the curves for $\gamma=0.008$ in Figs. 2(a)-2(c) of the original article. When we increased the precision to account for high levels of field amplification, no mode conversion was observed when the parameter loop was far away from the exceptional point (EP), which was mistakenly shown to be true as in Fig. 1(c) of the main text. In fact, for the parameter values listed there, the modal output should be $\left|\psi_{1}\right\rangle \rightarrow\left|\psi_{1}\right\rangle$ and $\left|\psi_{2}\right\rangle \rightarrow\left|\psi_{2}\right\rangle$ (corresponding to no mode conversion and no chirality).

This numerical error leaves the main message of our paper intact, which states that chiral mode conversion can also take place even without enclosing or encircling an EP in a dynamic contour. The results based on the increased numerical precision show, however, that chiral mode conversion requires the contour to be at least in the direct vicinity of the non-Hermitian degeneracy. To illustrate this, we consider a new loop that now lies closer to the EP and we compare the state conversion for two cycling rates $\gamma=0.5$ and $\gamma=0.1$. Specifically, in the upper panel of Fig. 1 presented below, the loop is centered at $\left(g_{0}, \rho\right)=(0.82,0.1)$ which excludes the EP. For the relatively nonadiabatic case, i.e., $\gamma=0.5$, no mode conversion was observed. Conversely, upon making the encirclement adiabatic, i.e., $\gamma=0.1$, both $\left|\psi_{1}\right\rangle$ and $\left|\psi_{2}\right\rangle$ (or any other linear combination of the two) end up in the same state $\left|\psi_{1}\right\rangle$, thus corroborating the findings of our analytical results. For the purpose of comparison, in this corrected Fig. 1, we also show the EP-inclusive case that depicts mode conversion both for small and fast rates of encirclement. Figure 2 of the original text should also be considered in the light of the aforementioned changes. Those results hold true only in the cases where

(a)

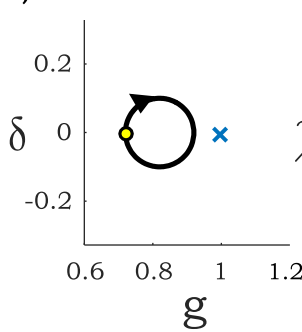

(d)

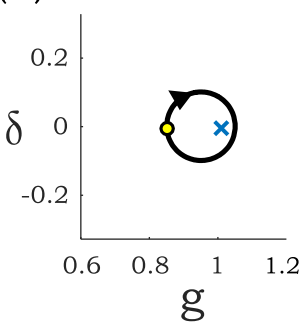

(b)

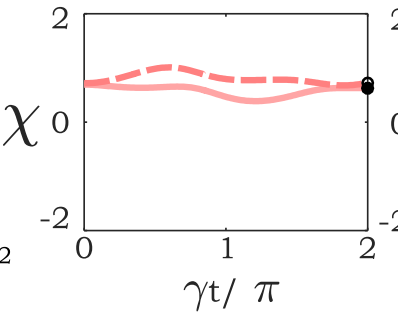

$\gamma=0.5$

(e)

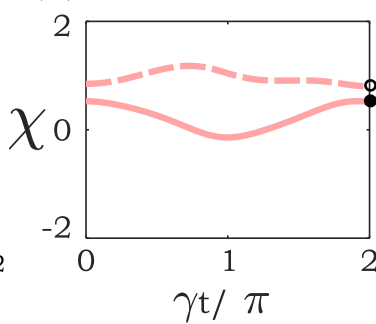

$\gamma=0.5$

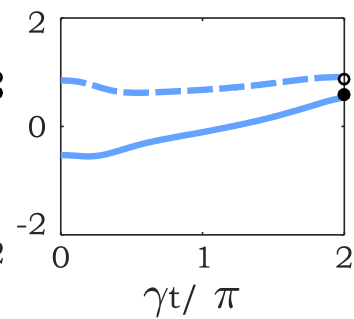

(c)

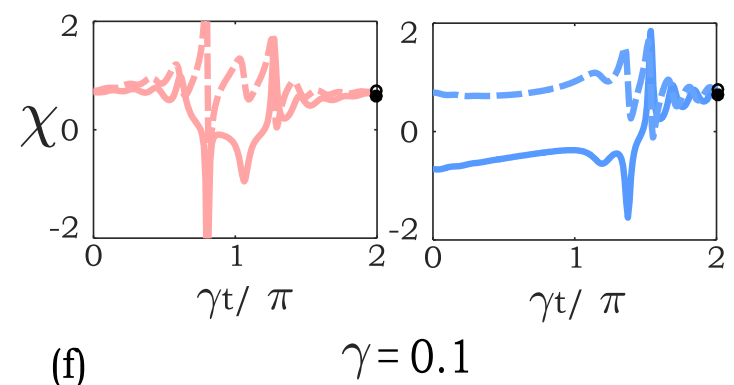

FIG. 1. Two different cw parameter cycles are shown in panels (a) and (d) along with the ensuing behavior of $\chi$ in the corresponding panels (b),(c) and (e),(f) in each row. The loop in panel (a) lies away from the EP (EP is shown as a cross) with $\left(g_{0}, \rho\right)=(0.82,0.1)$. In the one shown in panel (d), the contour includes the EP with $\left(g_{0}, \rho\right)=(0.95,0.1)$. The terminal points, where the two eigenvectors $\left|\psi_{1,2}\right\rangle$ are found, are marked by a yellow circle and the arrow shows the direction of encirclement. In panels (b) and (e), the resulting variation in $\chi$ at all times is shown when the rate of cycling is relatively large, i.e., $\gamma=0.5$. Plots on the left (shown in red) depict the case when the system is excited with $\left|\psi_{1}\right\rangle$ and those on the right (shown in blue) provide results for excitations with $\left|\psi_{2}\right\rangle$. In these plots, solid (dashed) lines represent real (imaginary) parts of $\chi$. As mentioned in the text, for this cw cycle, the state expected at the output is $\left|\psi_{1}\right\rangle$ that corresponds to $\chi \rightarrow e^{i \theta}$. The real (imaginary) part of this expected result is shown as a filled (empty) circle at $t=2 \pi \gamma^{-1}$. In the upper panels, these two circles lie very close to each other. In panels (c) and (f), the rate of cycling is reduced to $\gamma=0.1$ and both excitations end up at the correct location even for the non-EP enclosing case, panel (c). Although mode conversion is not robust in panel (b)—consider the plot on the right—results for the EP-inclusive loop show robust state conversion not only when the encirclement is slow [in panel (f)], but also when it is fast, $\gamma=0.5$, as in panel (e). 


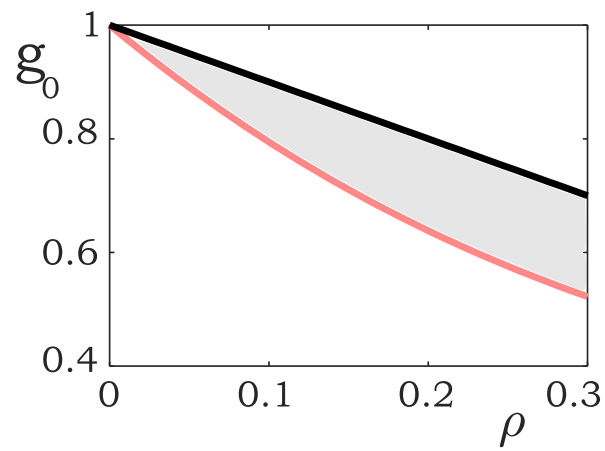

FIG. 2. Assuming adiabatic conditions (small values of $\gamma$ ), the shaded area shows the range of values of the loop center $g_{0}$ and radius $\rho$, for which chiral mode conversion can take place even without enclosing an EP. The red line is the curve obtained from Eq. (1) and the black line depicts the boundary where the loop starts touching the EP located at $\left(g_{0}+\rho=1\right)$.

$g_{0}$ and the radius of the circle $\rho$ satisfy the constraint given in Eq. (1) below. Furthermore, the curves for $\gamma=0.008$ also contain the numerical insufficiencies stated above.

Along these lines, we found that robust chiral mode conversion is not realized for any arbitrary loop in the $g-\delta$ plane, but takes place only if the loop is close to an EP. Our analysis suggests that as the loop center $g_{0}$ moves further away from the EP, the radius of this circle must be kept larger if mode conversion is desired. In this regard, we were able to obtain an approximate analytical form for this demarcation by requiring that the expression for $\eta$ [listed two lines above Eq. (6) of the original text] must be greater than zero, i.e., $\operatorname{Re}[\eta]>0$, so that $\left|e^{\nu \eta}\right| \gg\left|e^{-\nu \eta}\right|$. For small values of $z=x_{0} / v$, this latter requirement is satisfied provided that the following approximate expression holds true:

$$
\rho>2\left(\frac{1}{g_{0}}-g_{0}\right) e^{-2 \sinh ^{-1}(e / 2)},
$$

where $e$ is Euler's number. The region that excludes the EP but still gives mode conversion is depicted as shaded in Fig. 2 of this erratum. The analytical derivations that are presented in Eqs. (6)-(8) in the original text also rely on condition Eq. (1) to be fulfilled.

We also correct two typos in the original article: The correct Eq. (3) now reads

$$
\frac{d^{2} a(t)}{d t^{2}}=\left[\rho^{2} e^{2 i \gamma t}-\rho\left(2 g_{0}+i \gamma\right) e^{i \gamma t}+g_{0}^{2}-1\right] a(t) .
$$

In addition, regarding asymptotic expansions, the expression for $\eta$ has now been corrected to

$$
\eta=\sqrt{1+z^{2}}+\ln \left(\frac{z}{1+\sqrt{1+z^{2}}}\right) .
$$

We are indebted to our colleagues A. Schumer, Dr. J. Feilhauer, and Dr. S. Rotter from TU Wien, Austria, for pointing out the numerical deficiencies of our previous results and for helping us to find the restrictive region mentioned in Eq. (1) above. Their assistance and insightful discussions are greatly appreciated. 